\title{
Duelo perinatal: Un secreto dentro de un misterio.
}

Perinatal Grief: A secret within a mistery.

Ana Pía López García de Madinabeitia a .

${ }^{a}$ Enfermera especialista en Salud Mental. CSM “Gasteiz Centro”, de Vitoria-Gasteiz.

Correspondencia: Ana Pía López García de Madinabeitia (ana.pialopez@osakidetza.net)

Recibido: 12/04/2010; aceptado: 15/07/2010

RESUMEN: La muerte del feto durante el embarazo, en el parto o pocos días después del nacimiento constituye un tema delicado, condicionado por numerosos factores. Esta pérdida puede desencadenar reacciones de duelo en los progenitores y situaciones de difícil manejo para los profesionales sanitarios. Son duelos que reciben escasa consideración y que pueden complicarse dando lugar a trastornos psiquiátricos. Es necesario conocer el significado de la pérdida perinatal desde la perspectiva de los progenitores para no caer en posturas paternalistas o en protocolos dogmáticos que consideran iguales a todos los progenitores ante pérdidas a las que ellos atribuyen significados diferentes.

PALABRAS CLAVE: Pérdida, tabú, duelo, aborto, muerte fetal, mortinato.
ABSTRACT: Fetal death in pregnancy, during delivery or in the days after birth is a sensible topic, influenced by several factors. The loss can trigger grief reactions in parents as well as situations difficult to manage by health care workers. This grief, which receives scant consideration, can complicate and give rise to psychiatric disorders. It is necessary to know the significance of perinatal loss from the parents' perspective not to make the mistake of displaying paternalist attitudes or relying on dogmatic guidelines that consider all parents equal in the face of losses to which they attach different meanings. KEY WORDS: Bereavement, taboo, grief, miscarriage, fetal death, stillbirth.

\section{Introducción}

Durante siglos, tener descendencia ha sido un hecho natural. En la sociedad actual, en cambio, las maneras de lograr un embarazo se han ampliado debido a avances tecnológicos y además el concepto vario de familia se ha transformado dando lugar a otras variantes, generando nuevas formas de parentalidad.

Además, los avances en Ginecología y Obstetricia permiten ver la imagen ecográfica del hijo en gestación desde el comienzo del embarazo, lo que facilita el apego de los progenitores con el hijo que esperan. Pero este gran avance también les permite saber precozmente si el feto presenta problemas abocándoles a tomar decisiones de, literalmente, vida o muerte sobre su hijo y la continuidad del embarazo. Para los progenitores no es el peso, ni la edad gestacional lo que transforma al feto en hijo y lo dota de una identidad propia, sino que es el lugar que le han creado en su corazón, en su mundo afectivo, lo que condiciona que lo consideren hijo y persona. En palabras 
de von Raffler-Engel (1), "un aborto espontáneo o provocado no es un trozo de carne sin vida, sino un rudimentario ser humano que ha perdido la vida".

En España, en 2009, asociaciones como Umamanita, creada para apoyar a padres que han sufrido una pérdida perinatal, propusieron modificar la Ley de 8 Junio de 1957, sobre el Registro Civil para reflejar la filiación y otorgar nombre al feto nacido muerto o al nacido vivo que no ha superado las 24 horas. Actualmente es simplemente "el feto, varón o hembra difunto" de la mujer que lo parió. La proposición de ley llegó al Congreso de los Diputados pero en Junio de 2009 fue rechazada para decepción de sus promotores, que trataban de dar un reconocimiento emocional, que no jurídico, a los hijos que no llegaron a tener.

Por su parte, las normas de la Policía Sanitaria Mortuoria califican a los fetos muertos como "restos humanos de entidad suficiente procedentes de abortos" (2). A su vez, Barandiarán y Manterola (3) explican que en zonas rurales del País Vasco y Navarra los nacidos muertos o los muertos sin bautizar se colocaban bajo el alero de la casa o en un terreno contiguo, incluso dentro de la casa, en la cocina, el establo o la huerta. Se creía que los niños muertos sin bautizar iban al Limbo, lugar donde "ni se sufre, ni se padece, ni se tiene alegría" y se daba ese nombre a una parte del cementerio sin bendecir, no sagrada que solía tener una puerta de acceso directo, sin comunicación con el resto del cementerio. El cuerpo del bebé se envolvía en un lienzo o se colocaba desnudo en una caja de madera o cartón. El entierro se realizaba sin oficio religioso, ante pocos familiares y en ausencia de la madre, y no se rezaba ningún responso. Prácticas similares se realizaban en Irlanda, como indica Prior (4), donde el valor social de los difuntos se expresaba simbólicamente en la organización social del cementerio y la fosa común, utilizada para enterrar fetos muertos, era una tumba sin nombre que simbolizaba su nulo valor social.

Las actitudes hacia la pérdida perinatal varían según las expectativas de los progenitores, la prosperidad de la sociedad en que viven, y la cultura a que pertenezcan. Por ejemplo, en un pequeño estudio sobre mujeres hindúes de clase media se observaron reacciones intensas de duelo mientras sus familias las culpaban por no producir un bebé sano (5). Otro estudio en Taiwan destacaba la importancia cultural de la "ideología de continuidad", por la que se espera que la mujer sea capaz de aportar un hijo varón a la familia política (6). La mayoría de religiones conceden escasa atención a las pérdidas perinatales, y no hay rituales concretos (Tabla 1).

Este artículo pretende destacar la realidad del duelo perinatal, que como el duelo por otras personas queridas, desencadena un sufrimiento psicológico y puede complicarse convirtiendo la existencia de los progenitores sin hijo en una experiencia difícil de sobrellevar. 
ORIGINALES Y REVISIONES

Tabla 1

Las religiones ante la muerte del feto.

\begin{tabular}{|l|l|}
\hline - Catolicismo: & $\begin{array}{l}\text { El bautismo es para bebés vivos. Fetos mayores de } 24 \text { semanas, se permite entierro o } \\
\text { incineración, sin ceremonia ni bendición, en Limbo del cementerio, lugar desacralizado, en } \\
\text { fosa sin nombre. }\end{array}$ \\
\hline - Hinduismo: & $\begin{array}{l}\text { Se ata un cordón alrededor del cuello o la muñeca como señal de bendición. Al no reconocer } \\
\text { su vida, no hay pecado, ni necesidad de purificación por cremación. }\end{array}$ \\
\hline - Judaismo: & $\begin{array}{l}\text { Los no-judíos deben evitar tener contacto con el cuerpo. No hay rituales a menos que haya } \\
\text { vivido } 30 \text { días, pero se considera beneficioso para los padres rezar una oración. Cremación } \\
\text { prohibida. }\end{array}$ \\
\hline - Islamismo: & $\begin{array}{l}\text { El feto no tiene pecado; por lo que regresa directamente a Alá y facilita el camino de los } \\
\text { padres hasta Él. A partir de } 4 \text { meses de gestación se le da nombre, se lava, envuelve en } \\
\text { sábana y se entierra con opción a funeral. Cremación prohibida. }\end{array}$ \\
\hline - Budismo: & $\begin{array}{l}\text { Si feto mayor de 4-5 meses es un ser humano y puede reencarnarse. Con los fetos abortados } \\
\text { se celebra el misuko kuyo para pedirle perdón por haber perdido el embarazo y que no traiga } \\
\text { desgracia a la familia. }\end{array}$ \\
\hline
\end{tabular}

El duelo

"El dolor del duelo es tan parte de la vida como lo es la alegría del amor; es, quizás, el precio que pagamos por el amor, el coste del compromiso". Colin Murray Parkes (1986) (7)

Numerosos autores (Freud, Lindemann, Bowlby, Kübler- Ross, Murray-Parkes, Worden, Neimeyer, Rando, Stroebe) se han interesado desde el siglo pasado por el duelo, formulando teorías sobre las fases, el trabajo, o las etapas del mismo. El DSM-IV-TR define al Duelo (Z63.4) (8) como una reacción a la muerte de una persona querida, en la que algunos individuos afligidos presentan síntomas característicos de un episodio de depresión mayor, como tristeza, insomnio, anorexia y pérdida de peso. Sin embargo, la persona en duelo valora su ánimo depresivo como "normal". La duración y la expresión de un duelo "normal" varían considerablemente entre los diferentes grupos culturales. Desde una perspectiva más amplia se puede definir al duelo como una reacción y adaptación psicológica ante la pérdida de algo apreciado, vinculado al individuo: personas, objetos, mascotas, ilusiones, proyectos, etc. Es una experiencia única que cada persona afrontará con su propio estilo y recursos. Se asienta sobre una personalidad previa y se produce en unas circunstancias determinadas, y en un encuadre social concreto que condicionan el proceso y trabajo de duelo posterior. Las situaciones de duelo pueden repetirse de formas diversas a lo largo de la vida de una persona y resulta arriesgado concretar cuándo un duelo sigue un curso esperable y normal, o cuándo la sintomatología sugiere una patología psiquiátrica. Ciertas normas sociales tácitas orientan sobre quién puede y debe hacer el duelo, cómo, por quién y durante cuánto tiempo. Salirse de esos condicionamientos hace 
que algunos dolientes sufran la pérdida en soledad y sin apoyos al no verse autorizados para expresar lo que sienten, ni encontrar una escucha adecuada a su malestar.

El duelo tiene su propio conjunto de elementos (Tabla 2), destacando los síntomas psicológicos y afectivos, que pueden confundirse o solaparse con una depresión mayor, aunque ambos cuadros se distinguen por criterios sintomáticos y de duración del episodio. Ciertos síntomas depresivos no se observan en el duelo (8) como la culpa por cosas diferentes que las acciones realizadas o no por el superviviente en el momento de morir la persona querida, los pensamientos de muerte más que voluntad de vivir, el sentimiento de inutilidad, el enlentecimiento psicomotor acusado y prolongado o las experiencias alucinatorias distintas a escuchar la voz o ver la imagen fugaz de la persona fallecida. Y aunque la depresión es una de las complicaciones posibles del duelo (Tabla 3) (9), no debe diagnosticarse salvo que los síntomas se mantengan dos meses después de la pérdida. Sería útil contar con una definición más precisa o con instrumentos de medida adecuados para valorar el duelo transversal y longitudinalmente, pero las herramientas diseñadas se han limitado a la investigación clínica (10-12)

Tabla 2

Manifestaciones del duelo

\begin{tabular}{|l|l|}
\hline - Físicas: & $\begin{array}{l}\text { vacío en el estómago, opresión en pecho y garganta, ahogo, palpitaciones, suspiros, alteraciones } \\
\text { sueño, pesadillas, anergia, desgana sexual, apetito escaso o ansioso, pérdida ponderal, boca } \\
\text { seca, indigestión, cefaleas, sensibilidad al ruido, quejas somáticas. }\end{array}$ \\
\hline - Emocionales: & $\begin{array}{l}\text { insensibilidad, aturdimiento, tristeza, culpa, reproche, rabia, desesperación, hostilidad, } \\
\text { irritabilidad, anhedonia, soledad, vacío, desamparo, añoranza, alivio. }\end{array}$ \\
\hline - Conductuales: & $\begin{array}{l}\text { funcionamiento automático, mente ausente, aislamiento social, crisis de llanto, conductas de } \\
\text { búsqueda o evitación, objetos de apego, consumo tóxicos, hiperactividad, momificación. }\end{array}$ \\
\hline - Psicológicas: & $\begin{array}{l}\text { incredulidad, negación, rumiaciones sobre fallecido, confusión, irrealidad, ideas } \\
\text { suicidio/sustitución, sensación de presencia, idealización/ envilecimiento, alucinaciones e } \\
\text { ilusiones, falta concentración/ memoria. }\end{array}$ \\
\hline - Espirituales: & búsqueda de sentido, conciencia propia mortalidad, replanteamiento de creencias. \\
\hline
\end{tabular}

Tabla 3

Complicaciones posibles del duelo (7)

\begin{tabular}{|l|l|}
\hline - Físicas: & $\begin{array}{l}\text { Modificaciones de la respuesta inmune, activación adrenocortical (úlcera, HTA), incremento } \\
\text { de la prolactina sérica (con desarreglos menstruales), elevación de la hormona crecimiento } \\
\text { (con diabetes, HTA), mortalidad por problemas cardiacos. }\end{array}$ \\
\hline $\begin{array}{c}\text { - Psicológicas/ } \\
\text { Psiquiátricas: }\end{array}$ & $\begin{array}{l}\text { Depresión/ manía, reacción esquizofreniforme, cuadros de ansiedad y fobia, síndrome de } \\
\text { stress postraumático, trastornos de conducta, abuso de tóxicos. }\end{array}$ \\
\hline
\end{tabular}


ORIGINALES Y REVISIONES

\section{El duelo perinatal}

Cuando se produce una pérdida durante el embarazo, la vida y la muerte caminan juntas. Es una paradoja para la que nadie está preparado y por eso es tan delicado saber qué decir o hacer. Tampoco existen rituales religiosos que legitimen, faciliten y reconforten a los progenitores. Los familiares y amigos evitan hablar del tema por temor a causar más dolor que beneficio. Mientras, los padres viven su experiencia en soledad.

Aunque para la OMS (13) el período perinatal se extiende desde las 22 semanas de gestación (154 días) hasta una semana de vida independiente, en la práctica y en la literatura sobre el tema, este período se amplía. Kowalski (14) lo prolonga desde la concepción hasta el final del primer año de vida, lo que convierte en pérdidas perinatales al embarazo ectópico, al aborto espontáneo o inducido, a la reducción selectiva, a la muerte de un gemelo en gestación, al feto muerto intraútero o intraparto, a la muerte del prematuro, a la del neonato, y también a los bebés nacidos con anomalías congénitas o y los hijos cedidos en adopción (15). El concepto de pérdida perinatal rebasa el encuadre del periodo perinatal de la OMS e incluso de la pérdida por muerte.

Epidemiológicamente la mortalidad perinatal resulta de la suma de la mortalidad fetal tardía (marcada por la OMS en 28 semanas de gestación ó 1000 gramos) y la mortalidad neonatal precoz (hasta 7 días de vida), por 1000 nacidos (vivos o muertos). En España se considera muerte fetal tardía la del feto con 6 ó más meses de gestación y sólo se deben anotar en el legajo de abortos del Registro Civil las muertes fetales superiores a 180 días de gestación. Según el Instituto Nacional de Estadística, la mortalidad perinatal bajó del 10, 9 por mil de 1985 al 4,9 por mil de 2005 (16).

Esta revisión considerará pérdida perinatal la producida con resultado de muerte desde el conocimiento del embarazo hasta el periodo neonatal.

El silencio y el secretismo que rodean a la pérdida perinatal tienen que ver, entre otras cosas, con la intimidad. La mujer ha aprendido durante siglos a no hablar de aspectos como la menstruación, la sexualidad, la fertilidad, el embarazo o la menopausia más que en círculos privados y con otras mujeres. Estos fenómenos naturales se han visto envueltos en un halo de misterio y también ha sido esa la suerte de la pérdida del embarazo, que conjuga los tabúes de la sociedad actual sobre la muerte, el sexo y la reproducción.

\section{Literatura sobre la pérdida perinatal: una revisión}

El primer artículo sobre el sufrimiento de los progenitores apareció en 1959 (17). Recogía las dificultades del obstetra frente a la muerte fetal antes, durante y 
Tabla 4

Síntomas tras aborto espontáneo (32).

\begin{tabular}{|l|l|}
\hline - Físicos: & $\begin{array}{l}\text { Vacío en el estómago, Opresión en pecho y garganta, Dificultad respiratoria, Debilidad, } \\
\text { Fatiga, Sudoración. }\end{array}$ \\
\hline - Sentimientos: & $\begin{array}{l}\text { Shock, culpa, vacío, rabia, ansiedad, tristeza, reproche, confusión, incredulidad, desrealización, } \\
\text { despersonalización, soledad. }\end{array}$ \\
\hline - Pensamientos: & $\begin{array}{l}\text { Reexperimentación del trauma con ideas intrusivas y fantasías sobre el feto, movimientos } \\
\text { fetales "fantasma", alucinaciones auditivas y/o visuales con el feto, amnesia disociativa, } \\
\text { nivel de conciencia situacional disminuido, dificultades de concentración y toma de decisiones. }\end{array}$ \\
\hline - Conductuales: & $\begin{array}{l}\text { Dificultad para dormir, pesadillas, apetito escaso, aislamiento social, uso y abuso de sustancias, } \\
\text { evitación de situaciones sanitarias, mujeres embarazadas y niños, funcionamiento social y } \\
\text { laboral limitado. }\end{array}$ \\
\hline
\end{tabular}

después del parto, hacía recomendaciones humanistas sobre su abordaje y la atención a la pareja y mencionaba la pena del propio médico. Sugería informar a la madre acerca del parto y el bebé cuando despertase de la anestesia, pero no hablaba de verlo o tomarlo en brazos. Tanto si el hijo había muerto como si había nacido con malformaciones o discapacitado, aconsejaba recomendarle tener otro que le diera felicidad y confianza en sí misma.

En 1968, Bourne (18) abordó los efectos psicológicos del mortinato sobre las mujeres y sus médicos de cabecera en un artículo que se preguntaba por los motivos que hacían del mortinato una especie de "ángulo ciego" para los profesionales (professional blindspot), que pasaban por el trance simulando que no había ocurrido nada. Con intención seguramente protectora y beneficentista, ante una muerte perinatal no se daba ninguna posibilidad a los desolados progenitores de conocer a su hijo y poder así decirle hola y adiós. Los profesionales guardaban total silencio sobre lo ocurrido, se sedaba a la madre en el expulsivo para evitarle el dolor de ver a su hijo muerto y cuando despertaba, el cuerpo de su hijo ya había desaparecido de su vista (pero no de su corazón). Según Bourne se trataba como un "no-suceso", algo olvidable de inmediato. Para remediar su pena se recomendaba a los progenitores olvidar lo sucedido y buscar un nuevo embarazo cuanto antes.

Un estudio posterior de Kennell y colaboradores, (19) sobre duelo por neonatos, destacaba en 1970 como elementos distintivos la tristeza, el apetito escaso, la dificultad para dormir, la irritabilidad, la preocupación con el hijo perdido y la incapacidad para volver a la actividad normal. En 1976, Benfield y colaboradores (20) estudiaron las respuestas de duelo en progenitores de neonatos en riesgo vital y ese mismo año, Lewis publicó un trabajo sobre el manejo específico del mortinato (21). En 1980, Peppers y Knapp (22) propusieron otros nueve elementos del duelo perinatal: dificultad de concentración, rabia, culpabilidad, negativa a aceptar la realidad, confusión temporal, agotamiento, falta de energía, depresión y sueños repetitivos con el bebé perdido. 
ORIGINALES Y REVISIONES

En 1982 Kirkley-Best y Kellner (23) criticaron la bibliografía previa por sus errores metodológicos concluyendo que "sin un estudio apropiado, los profesionales están destinados a seguir las modas de los libros populares sobre duelo, sin llegar a cubrir las necesidades específicas de los padres de los mortinatos". Lo denominaron el duelo olvidado. Recomendaban que los progenitores vieran y tomaran en brazos al bebé muerto y proponían desarrollar grupos de apoyo para afectados. También en 1982 Callahan y colaboradores (24) plantearon que la pérdida perinatal es distinta de otras por la "reticencia del sistema de apoyo a hablar de lo ocurrido, porque muchas veces no se conoce el motivo de la muerte y por la culpabilidad intensa que genera". En 1984, Herz (25) añadió otros matices como que la mujer se siente traicionada por su propio cuerpo, experimenta la rabia de tener que sustituir un acto que debería haber sido alegre por otro traumático (funeral), o la envidia hacia las personas que han culminado el embarazo con éxito, presenta sentimientos de vulnerabilidad y pérdida de control, tiene la sensación de haber decepcionado a su pareja y está expuesta a la posibilidad de tener múltiples reacciones de aniversario (fecha prevista de parto, fecha de la pérdida, fecha de noticia del embarazo). La mayoría de los artículos sobre la repercusión de la pérdida perinatal se interesan por la madre o por ambos progenitores, pero, a medida que el padre va teniendo más presencia en el seguimiento del embarazo y el parto, también va creciendo el interés por estudiar sus reacciones a la pérdida. $(26,27)$.

La investigación va reconociendo los rasgos especiales del duelo perinatal, por las múltiples pérdidas que supone para los progenitores (el hijo proyectado, aspectos de sí mismos, regalos y atenciones, una etapa de la vida, un sueño y una creación) (28). Pese a la tragedia que sufren, se ven inmersos en un duelo desautorizado en términos de Doka (29), ya que se trata de una pérdida que no puede ser abiertamente reconocida, expresada en público o apoyada por la red social. Tales duelos tienen lugar cuando la relación se deslegitima (extramarital, de convivencia, homosexuales, ex cónyuges, amigos del pasado, etc), cuando las circunstancias de la muerte generan vergüenza o disgusto y el afectado teme sentir cierto reproche social (SIDA, suicidio, sobredosis) y cuando la pérdida no es reconocida, como en las perinatales, las de padres que dan a sus hijos en adopción o tras acogimiento, o las de mascotas.

\section{Tipos de pérdida perinatal}

\subsubsection{Aborto espontáneo}

Se define como la terminación natural del embarazo antes de que el feto sea viable. Se estima que entre el 15 y el $25 \%$ de los embarazos terminan en aborto, la mayoría de los casos antes de las 12 semanas de gestación (30-31). Aunque no 
siempre resulta traumática, la pérdida puede acompañarse de fenómenos de duelo, de intensidad y duración relacionada con la edad gestacional. El final del embarazo por aborto es una pérdida no reconocida social ni sanitariamente. Si no hay vida, tampoco hay muerte, ni duelo.

Moscarello (32) describe los síntomas tras el aborto espontáneo (Tabla 4), a los que deben añadirse los sentimientos de ineficacia que asolan a las madres (33), y su sensación de ser imperfectas o inadecuadas para gestar un bebé (defectus incubus). Para Côte-Arsenault y Marshall (34) la mujer se siente traicionada por su propio cuerpo, como si algo fuera mal con su feminidad, generándose sentimientos de autorreproche y culpabilidad. Afronta su desazón comentando reiterada y detalladamente lo sucedido. Le agrada leer sobre duelo y sentimientos y espera la misma respuesta de su pareja, por lo que si no la obtiene cree que a él no le importa tanto como a ella lo ocurrido. Busca el apoyo grupal, se solidariza con experiencias similares y pierde interés por las relaciones sexuales.

Tras la pérdida del embarazo es incómodo explicar que ya no habrá bebé a los mismos a quienes recientemente se anunció el embarazo. También incomoda que quienes desconocen lo ocurrido pregunten por el embarazo supuestamente en curso. Las actitudes sociales y familiares tras un aborto reflejan una tendencia a disimular como si nada hubiera ocurrido. Se resta importancia a la pérdida, convirtiendo lo que para los progenitores era un bebé en un ser a medio hacer, con escasa condición humana y se augura como cierto que habrá nuevos embarazos en el futuro: "Sois jóvenes", "en realidad, no era un auténtico bebé", "la Naturaleza es sabia".

\subsubsection{Aborto voluntario}

Terminar un embarazo por voluntad propia también puede desencadenar una reacción de duelo que recibe una creciente atención en la literatura. Peppers fue el primero en documentar la respuesta de duelo tras aborto voluntario y encontró que las puntuaciones eran similares a las de mujeres que habían tenido un aborto espontáneo, un mortinato o una muerte neonatal (35). Si en abortos espontáneos parece haber una conspiración de silencio en su entorno social, en el aborto voluntario, el secretismo es aún mayor.

\subsubsection{Interrupción voluntaria de embarazo por problemas del feto o ame- naza para la salud materna}

Gran parte de estas pérdidas se produce cuando los padres saben que el feto porta alguna malformación o enfermedad. Las mujeres que toman la decisión de abortar se ven abrumadas por sentimientos de culpa y vergüenza, no sólo por haber gestado un feto defectuoso, sino por haber decidido su muerte. Surgen una serie de paradojas (36), como que la mujer vea al mismo tiempo la terminación del embarazo como un acto de amor y/o como un asesinato, sienta que ha perdido la oportunidad de ser madre y/o no se vea capaz de serlo de "ese" hijo, quiera ser absuelta por su decisión y/o sienta que no hay absolución humana suficiente o, finalmente, agradezca 
ORIGINALES Y REVISIONES

que la tecnología haya permitido conocer la realidad al tiempo que está resentida porque esa tecnología ha forzado una decisión trascendente para ella y para la vida de su propio hijo en gestación. No es infrecuente camuflar esta pérdida voluntaria del embarazo como un aborto espontáneo.

\subsubsection{Reducción selectiva en embarazos múltiples}

Los embarazos múltiples suelen ser producto de técnicas de fertilidad, lo que implica que las parejas cuentan ya con una historia de pérdidas o frustraciones. Si la supervivencia de todos los fetos es inviable se practica una reducción entre las semanas 10 y 12, por inyección letal. Es una amarga decisión que hay que tomar contra el reloj, la energía, la ilusión y el dinero. Los progenitores encaran el impensable dilema de "cuántos son demasiados" después de años de ser incapaces de concebir. La reducción permite un mejor resultado del embarazo que, de haber continuado, podría haber acabado con prematuridad extrema, discapacidad severa o la muerte de todos los fetos. Apenas se han estudiado las consecuencias psicológicas para la pareja y menos aun para los hijos supervivientes. Además de por la pena, el dolor crece por la incomprensión y soledad pues la mayoría lleva lo ocurrido en secreto, sin dar explicaciones a su entorno para evitar ser enjuiciados. Aunque muchos progenitores no sufren una pena prolongada un tercio de ellos presenta una depresión moderada (37) y no pocas mujeres recuerdan la intervención como atemorizante, muy estresante y causante de dolor emocional. Muchas parejas se preguntan cómo habrían sido los hijos que no llegaron a gestar, cómo se hubieran arreglado de haber tenido trillizos, o si los supervivientes echarán de menos durante su vida al hermano con quien compartieron útero.

\subsubsection{La pérdida por feto muerto intraútero, intraparto (mortinato)}

La muerte fetal, ya sea intraútero o en el parto desencadena, según Stroebe y Schut (38), varias pérdidas que funcionan como estresores. La principal es el bebé ansiado, pero hay otras pérdidas colaterales importantes:

- el momento de convertirse en padre o madre,

- el rol de padre o madre si es el primer hijo,

- la composición familiar como se imaginaba,

- el reconocimiento de ese hijo en la mente de los demás pese al tiempo transcurrido

- la confianza en la seguridad de otros hijos,

- la inocencia respecto al embarazo y parto,

- los amigos o familiares que no estuvieron a la altura y negaron la relevancia de la pérdida,

- el derecho a mencionar ese hijo en ciertos lugares

- el contacto y la posibilidad de crear recuerdos

\subsubsection{Pérdida en embarazos múltiples}

La mortalidad perinatal en embarazos múltiples es 7- 8 veces superior a la del 
embarazo único (39). Las expectativas de los progenitores y el interés de los allegados convierten a esta gestación en especial. Por este motivo, cuando uno o más fetos fallecen, la pareja no sólo pierde un bebé, sino un gemelo. Si la madre debe continuar el embarazo con un feto vivo y otro muerto, necesitará todo el apoyo y comprensión del personal sanitario. El momento del parto será temible y debería darse a los progenitores la oportunidad de tomar en brazos a los dos bebés, si lo desean, sin verse forzados a hacerlo y previa descripción del estado del bebé muerto. Divididos entre la pena por el bebé perdido o moribundo y la alegría por el superviviente, que se convierte en el recuerdo interminable de lo que podría haber sido el otro (bautizo, cumpleaños, el primer diente), sentirán siempre añoranza por el gemelo al que no tuvieron la oportunidad de conocer y a quien no verán crecer (40).

\subsection{La pérdida del neonato}

Según Pallás y de la Cruz (41), "actualmente se tienen expectativas muy altas con respecto a las unidades de cuidados intensivos, se tiende a pensar que todos los niños pueden salir adelante. La realidad, sin embargo, es bien diferente". Algunos problemas graves del bebé, que comprometen su supervivencia, se anticipan durante el embarazo y no resultan sorpresivos para los padres ni el equipo. Otros, sin embargo, aparecen inesperadamente durante el parto o en las primeras horas o días de vida del neonato. En ambos casos los progenitores y los profesionales se enfrentan a una situación que podría considerarse de duelo anticipatorio (42) dada la gravedad y riesgo de muerte del bebé. Por un lado elaboran la pérdida del bebé sano fantaseado y, por otro, anticipan la posible pérdida por muerte del bebé real. Se debaten entre el apego al bebé enfermo y el temor a que no logre sobrevivir. Esta ambivalencia puede manifestarse por la distancia emocional e incluso física del bebé o por una constante presencia y alerta por todo lo que sucede con su hijo. Su vida cotidiana está condicionada por las visitas hospitalarias y la evolución del estado del niño. La situación puede llegar al punto de desear que el bebé muera y así acabar con el sufrimiento de todos, aunque ese pensamiento conlleva un intenso sentimiento de culpa. Cuando los profesionales plantean limitar el esfuerzo terapéutico los progenitores se ven enfrentados a una dramática decisión.

\section{Duelo perinatal: Aspectos clínicos}

Para Mander (43) el duelo perinatal tiene unas características que lo diferencian de duelos de otros tipos: la proximidad entre el nacimiento y la muerte, la juventud de los progenitores para los que supone su primer contacto con la muerte y lo inesperado del suceso. Hay que añadir que se trata de la pérdida de una relación más simbólica que real basada en sus necesidades y deseos (44). 
La experiencia clínica demuestra que, tras la pérdida perinatal, la persona en duelo experimenta shock e insensibilidad, aturdimiento y dificultades para funcionar con normalidad. Siente añoranza y tiene conductas de búsqueda, con irritabilidad, labilidad, debilidad y sentimientos de culpa. Algunas mujeres refieren oír el llanto del bebé o sentir sus movimientos en el vientre. Aparece posteriormente la desorientación y desorganización de la vida cotidiana, con sensación de vacío y desamparo. Se sienten desautorizados para estar en duelo, temen enfermar y deprimirse. Estos fenómenos comienzan cuando todo el mundo se sorprende de que no lo haya "superado", pues "hay que seguir adelante"... y tener otro hijo. Finalmente se produce una reorganización, en la que sin olvidar la pérdida, se rehace la vida y se recupera la capacidad de disfrutar

Ronald Knapp (33) distingue las siguientes modalidades de duelo de progenitores por sus hijos:

- Promesa de no olvidar nunca al hijo, reivindicando su existencia, frente a la presión del entorno para que lo olviden

- Deseo de morir y estar unidos en la muerte con el hijo

- Revitalización de creencias religiosas, buscando un sentido a la pérdida

- Cambio de los valores en la vida cotidiana, dando mayor importancia a los pequeños detalles

- Mayor tolerancia y mejor comprensión del sufrimiento de otros

- La "sombra del duelo", descrita como "una forma de duelo crónico que a veces arrastran durante la mayor parte de sus vidas", "un dolor sombrío subyacente a los sentimientos de la persona". Se observa más en las madres, quienes "no pueden dejar de recordar la pérdida sin sentir algún tipo de emoción, aunque sea muy débil"

En cualquiera de sus variantes el duelo puede complicarse con síntomas psiquiátricos si concurren algunos factores de riesgo (Tabla 5) (9). Para el duelo peri-

Tabla 5

Factores de riesgo de duelo complicado.

\begin{tabular}{|l|l|}
\hline \multicolumn{1}{|c|}{ Aplicables a todo tipo de duelos } & \multicolumn{1}{c|}{ En el duelo perinatal $(45,46)$} \\
\hline Circunstancias especiales de la muerte: repentina, & Problemas psiquiátricos previos \\
inesperada, violenta, en una catástrofe, sin cadáver o & No tener hijos \\
sin poder verlo, vergonzante, acumulativa & No tener familia ó apoyo social \\
Enfermedades físicas y mentales & Escasa información sobre los abortos \\
El muerto es un niño & No haber explicación para lo sucedido \\
Red de apoyo escasa o ineficaz & Pérdidas recurrentes \\
Duelos previos no resueltos & Historia actual de depresión \\
Relación ambivalente o sobredependiente con el & No tener pareja \\
fallecido & Coincide con otros problemas vitales importantes \\
Recursos personales escasos & \\
\hline
\end{tabular}


natal, Neugebauer (45) y Athey (46) han identificado como factores de riesgo los antecedentes de problemas psiquiátricos previos, una historia de pérdidas recurrentes, la presencia de depresión, la falta de hijos, pareja estable, familia o apoyo social, disponer de escasa información sobre los abortos, la inexistencia de una explicación para lo sucedido o, finalmente, que la pérdida coincida con otros problemas vitales importantes.

En el duelo tras aborto espontáneo es determinante que la pérdida haya tenido lugar en una fase relativamente avanzada del embarazo, cuando se perciben los movimientos fetales. También influye que sea el primer embarazo, que el hijo sea deseado, programado o no, que se trate de una gestación al final de la vida reproductiva o fruto de técnicas de fertilización, y finalmente factores psicosociales y dependientes de la personalidad y la cultura (47). El duelo perinatal puede dar lugar a trastornos psiquiátricos como:

- Trastornos depresivos: entre el 10 (48) y el 48\% (49). Son más frecuentes en mujeres con antecedentes depresivos o psiquiátricos en general, nulíparas o que han tenido abortos previos. Deben tenerse presentes los rasgos que permiten el diagnóstico diferencial entre la depresión y el duelo (Tabla 6) (50)

- Trastornos de ansiedad: aumentan tras el aborto para disminuir después de las 12 semanas (51), pero sobre todo despuntan ante la posibilidad de un nuevo embarazo $(25 ; 52-54)$

- Trastorno por stress postraumático: entre el 2 y $5 \%$ en partos instrumentales y cesáreas , y hasta el $25 \%$ en el mes posterior a la pérdida y hasta el $7 \%$ cuatro meses después $(55,56)$.

Existen varias escalas para valorar el duelo perinatal. El PGS (Perinatal Grief Scale) (57), la MGS (Munich Grief Scale) (58), el TGI (Texas Grief Inventory) adaptada para aborto (59), la PGS (Perinatal grief scale) (60) o la PBS (Perinatal Bereavement Scale) (61).

Tabla 6

Diferencias entre duelo perinatal y depresión.

\begin{tabular}{|l|l|}
\hline Duelo & Depresión \\
\hline Evocan tristeza y empatía & Evocan distancia, impaciencia o irritación \\
\hline Preocupación por el hijo perdido & Aislamiento y autocompasión \\
\hline Pena al ver bebés y embarazadas & Recuerdos desagradables sobre el embarazo \\
\hline Crisis de llanto & Idealización del feto muerto \\
\hline Deseo de hablar de lo sucedido & Vergüenza y desvalorización \\
\hline $\begin{array}{l}\text { Autorreproche limitado a creer no haber hecho lo } \\
\text { suficiente por el feto }\end{array}$ & Sensación de haber fracasado como mujer \\
\hline Ideas de suicidio no son frecuentes & Ideas de suicidio frecuentes \\
\hline Búsqueda de consuelo en otras personas & Abandono de relaciones sociales \\
\hline Dolor por la pérdida & Desesperanza, desconsuelo \\
\hline Soledad y vacío & Culpabilidad global \\
\hline
\end{tabular}


ORIGINALES Y REVISIONES

\section{Los otros afectados}

Aunque la mayoría de los artículos sobre la repercusión de la pérdida perinatal colocan a la madre como la más afectada, el duelo de otros miembros de la familia merece igualmente consideración.

Madres y padres desarrollan un apego diferente. El apego materno puede aparecer mucho antes de confirmarse el embarazo, desde que la mujer fantasea con la maternidad, y aumenta cuando nota moverse al feto en su vientre. El apego paterno es más intelectual que emocional o físico. Estar presente en la ecografía le vincula realmente con el hijo que esperan. Este "vínculo incongruente" (62) entre padre y madre genera diferencias en el apego con el feto y, si éste muere, el duelo de cada uno tendrá distintos estilos y ritmos (63).

El padre se pregunta por el motivo de lo ocurrido, cómo consolar a su pareja y si podrán tener otro hijo en el futuro. Su reacción viene dictada por las responsabilidades que debe asumir. Se espera que apoye a la madre física y emocionalmente, al tiempo que es quien debe informar de lo sucedido a familia y amigos y preparar el entierro del bebé. En el varón es común un sentimiento de desbordamiento por la pena de su esposa, que se impone a la expresión del duelo propio. Más enfadado que culpabilizado, dirige su rabia hacia el personal sanitario, se vuelca en su trabajo, en la hiperactividad y en los cambios en sus rutinas, y siente su pena en secreto y en solitario (64). Cuando se repara en él es para preguntarle por su pareja, como si fuera un mero acompañante (65). Se siente incómodo cuando se le pregunta por sus sentimientos y evita confrontaciones que evidencien sus emociones. No busca ayuda aunque la necesite y sepa que debe confiar su pena a alguien, preferiblemente otro varón (26). Puede plantear a su pareja tener sexo como cercanía e intimidad, lo que puede verse como inadecuado e interpretarse como una muestra de insensibilidad. Puesto que ambos tienen su propio ritmo y manera de afrontamiento, no es rara la aparición de conflictos de pareja (66).

Los hermanos sufren también la pérdida, aunque relegados del proceso de duelo son los "dolientes olvidados" (67). Su reacción depende de la edad, sexo, personalidad, lugar en la fratria, y la cultura y religión familiares. Pueden culpabilizarse por creerse causantes de la pérdida por sus sentimientos de envidia y rabia al verse desplazados en el cariño de los progenitores por el bebé esperado. Si no han llegado a conocer el embarazo, los progenitores deberán informarles del motivo de su disgusto, insistiendo en que no van a morir como su hermanito ni les perderán también a ellos, buscando tranquilizarles y liberarles de posibles ideas de culpa o responsabilidad por lo sucedido. Es necesario responder honestamente a sus preguntas, con sencillez y sin mentiras, y hacerles partícipes de los rituales de despedida del bebé a través de dibujos, flores o pequeños regalos (68)

Los nietos ayudan a los abuelos a fortalecer los vínculos con sus hijos, por lo que una pérdida perinatal les hace sufrir doblemente: por el nieto perdido y por el hijo 
en duelo. Pueden aparecer deseos de sustituir al nieto perdido, al que ven como una vida desperdiciada frente a la suya, ya vivida. Los abuelos no deberían tomar decisiones por sus hijos sin su autorización, aunque pueden ayudar en tareas domésticas y el cuidado de otros nietos. La pérdida de un nieto posibilita el duelo por antiguas pérdidas de embarazos propios y acerca a la madre en duelo reciente a su propia madre (68, 69).

\section{Conclusiones}

En esta revisión hemos pretendido hacer visible el duelo perinatal, que en España cuenta con una escasa consideración social y sanitaria. Hace apenas 50 años se empezó a plantear en otros países la conveniencia de modificar las prácticas paternalistas hacia la madre, negligentes para con el padre y evitadoras con el feto. Surgieron protocolos y guías de actuación para orientar a los profesionales sanitarios en el abordaje de las situaciones de muerte fetal. Algunas de ellas más que orientar se reducían a un listado de tareas que cumplir y se llegó a confundir un medio con un fin en sí mismo. Para Irving Leon (70), estas prácticas, que tuvieron su mayor apogeo en las décadas de los 80 y 90, tienen cuatro riesgos: la institucionalización de la pérdida, la idealización del contacto con el feto muerto, la homogeneización del luto, y el aleccionamiento a los padres sobre cómo deben vivir el duelo. Considera este autor que "las respuestas de los cuidadores a la pareja en duelo se han convertido "en una puesta en escena muy cuidada, marcada por un guión, en lugar de lo que debería ser una comunicación genuina entre los afectados y el equipo que les atiende".

Cada persona es diferente, como lo es su apego con el recién nacido o el no nacido, y también lo es su manera de afrontar la pérdida. Por lo tanto, el aprendizaje de los profesionales para desenvolverse en estas situaciones tiene que ver con saber estar, escuchar y comprender la pena ajena. Sin embargo, hay que ser conscientes de que la intervención hospitalaria es muy recortada, y que en la mayoría de los casos solo da tiempo a prestar unos "primeros auxilios psicológicos" (71), que no eliminan el dolor de la pérdida pero sí facilitan el comienzo del proceso de duelo. El equipo hospitalario deberá gestionar que tras el alta los progenitores tengan unos cuidados físicos y emocionales que den continuidad a los iniciados durante el ingreso para poder asimilar progresivamente lo vivido y seguir adelante con su vida. Los profesionales deben saber que "los padres nunca olvidan la comprensión, el respeto y el calor genuino que recibieron de los cuidadores, que llega a ser tan duradero e importante como los recuerdos materiales del embarazo perdido y de la corta vida de su bebé" (72). 
ORIGINALES Y REVISIONES

\section{BIBLIOGRAFÍA:}

(1) Von Raffler-Engel W. The perception of the unborn across the cultures of the world. Toronto: Hogrefe \& Huber Publishers. 1994

(2) SeoAne JA. El destino de los fetos muertos en los hospitales. Apuntes sobre la situación jurídica en el País Vasco. Comunicación personal. 14 Junio 2003

(3) Barandiarán JM, Manterola A. Atlas etnográfico de Vasconia. Ritos del nacimiento al matrimonio. Bilbao: Instituto Labayru, 1998

(4) PRIOR L. The social organization of death; medical discourse and social practices in Belfast. London: Macmillan, 1989

(5) Mammen OK. Women's reactions to perinatal loss in India: an exploratory, descriptive study. Infant Men Health J 1995; 16: 94-101

(6) Hsu MT, TsEng YF\& Kuo LL. Transforming loss: Taiwanese women's adaptation to stillbirth. J Adv Nurs 2002; 40: 387-395

(7) PARKES CM. Bereavement: Studies of grief in adult life, 2nd edition. London: Penguin Books, 1986

(8) American Psychiatric Association: DSM-IV-TR. Manual diagnóstico y estadístico de los trastornos mentales; $4^{\text {a }}$ Edición, Texto Revisado. Barcelona: Masson, 2003

(9) PARKES CM. Bereavement in adult life. BMJ 1998; 316: 856-9

(10) Faschingbauer TR, Devaul RA, Zisook S. Development of the Texas Inventory of grief. Am J Psychiatry 1977; 134: 696- 698

(11) Sanders CM, Mauger PA, Strong Pn. A Manual for the Grief Experience Inventory. Blowing Rock, NC: Center for Study of Separation and Loss, 1985

(12) Prigerson HG, Maciejewski PK, Reynolds CF. Inventory of complicated grief: a scale to measure maladaptive symptoms of loss. Psychiatry Res 1995; 59: 65-79

2006

(13) WHO. Neonatal and perinatal mortality: country, regional and global estimates Ginebra:WHO,

(14) Kowalski K. Perinatal loss and bereavement. En: Sonstegard L, Kowalski K, Jennings B, eds. Crisis and Illness in Childbearing (Women's Health), vol 3. New York: Grune and Stratton, 1987; pp: $25-42$

(15) Wallerstedt C, Higgins P. Perinatal circumstances that evoke differences in the grieving response. J Perinatal Educ 1994; 3: 35-39

(16) Instituto Nacional de Estadística. Movimiento natural de la población. Datos Europeos.

(17) Elia AD. The management of grief situations in obstetrics. BMQ 1959; 10: 6-12

(18) BouRnE S The psychological effects of stillbirths on women and their doctors. J R Coll Gen Pract 1968; 16: 103-112

(19) Kennell JH, Slyter H, Klaus MH. The mourning response of parents to the death of a newborn infant. N Engl J Med. 1970; 283: 344-9.

(20) BeNFieLd DC, Leib SA, RENTER J. Grief response of parents after referral of the critically ill newborn to a regional center. N EngI Med J 1976; $294: 975$

(21) LEwIS E. The management of stillbirth: coping with an unreality. Lancet 1976; 2(7986): 619-20

(22) PEPPERS LG, KNAPP RJ. Maternal reactions to involuntary fetal/infant death. Psychiatry 1980; 43: 155-9 
(23) Kirkley-Best E, Kellner KR. The forgotten grief: a review of the psychology of stillbirth. Am J Orthopsychiatry 1982; 52: 420-9

(24) Callahan EJ, Brasted WS, Granados JL. Life span developmental psychology. Non-normative life events. New York: Academic Press, 1983

(25) HERz E. Psychological repercusions of pregnancy loss. Psychiatr Ann 1984; 14: 454-457

(26) Samuelsson M, Radestad i, Segesten K. A waste of life: Fathers'experience of losing a child before birth. Birth 2001; 28: 124-130

(27) Badenhorst W, Riches S, Turton P, Hugues P. The psychological effects of stillbirth and neonatal death on fathers: Systematic review. J Psychosom Obstet Gynaecol 2006; 27: 245- 256

(28) KowalSki K. Perinatal loss and bereavement. En KR Simpson \& PA Creehan (Eds) Perinatal nursing. Philadelphia: Lippincott Williams \& Wilkins, 2001; pp 476- 491

(29) Dока K (EDITOR). Disenfranchised grief. New directions, challenges and strategies for practice. Champaing (Illinois): Research Press, 2002

(30) Hemminki E, Forssas E. Epidemiology of miscarriage and its relation to other reproductive events in Finland. Am J Obstet Gynecol 1999; 181: 396-401.

(31) CRAmer DW, Wise LA. The epidemiology of recurrent pregnancy loss. Semin Reprod Med 2000; 18: 331-9.

(32) MosCarello R. Perinatal bereavement support service: three-year review. J Palliat Care 1989; $5: 12-8$

(33) Knapp RJ. Beyond endurance. When a child dies. New York: Schocken Books, 1986

(34) Côté-Arsenault D, Marshall R. One foot in- one foot out: Weathering the storm of pregnancy after perinatal loss. Res Nurs Health 2000; 23: 473-85

(35) PEPPERs L. Grief and elective abortion: breaking the emotional bond? Omega 1987-1988; 18: $1-10$

(36) SLOAN EP. Viewing the fetus following termination of pregnancy for fetal anomaly. JOGGN 2008; 37, 395- 404

(37) Schreiner-Engel P, Walther NV, Mindes J, Lynch L, Berkowitz RL. First-trimester multifetal pregnancy reduction: Acute and persistent psychologic reactions. Am. J. Obstet. Gynecol 1995; 172: $541-552$

(38) Stroebe M, Schut H. Dual process model. En Neimeyer R (Ed) Meaning reconstruction and the experience of loss. Washington DC: American Psychological Association, 1999; pp 375-404.

(39) Chitrit Y, Filidori M, Pons JC, Duyme M, PAPIERniK E. Perinatal mortality in twin pregnancies: a 3 year analysis in Seine Saint- Denise (France). Eur J Obstet Gynecol Reprod Biol 1999; 86: 23-8

(40) SAmuels VR. Always my twin. Victoria, BC: Trafford, 2005

(41) Pallás CR, De, la Cruz J. Antes de tiempo. Nacer muy pequeño. Madrid: Ex libris, 2004

(42) Lindemann E. Symptomatology and management of acute grief. Am J Psychiatry 1944; 101: $141-148$

(43) MANDER R. Loss and bereavement in childbearing. Oxford: Blackwell Publishing, 1994

(44) BRIER, N. Grief following miscarriage: a comprehensive review of the literature. J Womens Health (Larchmt) 2008; 17: 451- 464

(45) Neugebauer R, Kline J, O’Connor P, Shrout, Johnson J, Skodol A, et al. Determinants of depressive symptoms in the early weeks after miscarriage. Am J Public Health 1992; 82: 1332-9

(46) Athey J, Spielvogel AM. Risk factors and interventions for psychological sequelae in wo- 
ORIGINALES Y REVISIONES

men after miscarriage. Prim Care Update Ob Gyn 2000; 7: 64- 69

(47) Boyce P M, Condon JT, Ellwood DA. Pregnancy loss: a major life event affecting emotional health and wellbeing. MJA 2002; 176: 250-251

(48) Neugebauer R, Kline J, Shrout P, Skodol A, O'Connor P, Geller PA. Major depressive disorder in the 6 months after miscarriage. JAMA 1997; 277: 383-8.

(49) Friedman T, Gath D. The psychiatric consequences of spontaneous abortion. Br J Psychiatry $1989 ; 155: 810-3$.

(50) Beutel M, DecKard R, von RAD M, Weiner H Grief and depression after miscarriage: their separation, antecedents and course. Psychosom Med 1995; 57: 517-526

(51) Prettyman RJ, CoRdle CJ, CoOK GD. A three-month follow-up of psychological morbidity after early miscarriage. Br J Med Psychol1993; 66: 363-72

(52) Armstrong D, Hutti M. Pregnancy after perinatal loss: the relationship between anxiety and prenatal attachment. J Obstet Gynecol Neonatal Nurs 1998; 27: 183-9.

(53) Côté-Arsenault D. The influence of perinatal loss on anxiety in multigravidas. J Obstet Gynecol Neonatal Nurs 2003; 32: 623-9.

(54) Geller PA, Kerns D, Klier CM. Anxiety following miscarriage and the subsequent pregnancy: a review of the literature and future directions. J Psychosom Res 2004; 56: 35-45.

(55) AyERS S. Post-traumatic stress following childbirth: a review of the emerging literature and directions for research and practice: commentary. Psychol Health Med 2003; 8: 169-171

(56) Brier N. Anxiety After Miscarriage: A Review of the Empirical Literature and Implications for Clinical Practice. Birth 2004; 31: 138-42

(57) TOEdter LJ, Lasker JN, AldahefF JM. The perinatal grief scale: development and initial validation. Am J Orthopsychiatry 1998; 58: 435- 449

(58) Beutel M, Will H, VölKL K, von Rad M, Weiner H. Entwicklung und Erfassung von Trauer am Beispiel des Verlustes einer Schwangerschaft: Die Miinchner Trauerskala (MTS). Psychother Psychosommed Psychol 1995; 45:295-302

(59) Nikcevic AV, Snidders R, Nicolaides KH, Kupek E. Some psychometric properties of the Texas Grief Inventory adjusted for miscarriage. Br J Med Psychol 1999; 72: 171- 178

(60) Hutti M, de Pacheco M, Smith M. A study of miscarriage: Development and validation of the Perinatal Grief Intensity Scale. JOGNN 1998; 27: 547-555

(61) Theut SK, Pedersen FA, Zaslow MJ, Cain RL, Rabinovich BA, Morihisa JM. Perinatal loss and parental bereavement. Am J Psychiatry 1989; 146: 635-9.

(62) Peppers L, Knapp R. Motherhood and mourning a perinatal death. New YorK: Praeger Publishers, 1980

(63) Menke J, Mc Clead R. Perinatal grief and mourning. Adv Pediatr 1990; 37: 261- 283

(64) Johnson MP, Puddifoot JE. The grief response in the partners of women who miscarry. Br J Psych 1996; 69: 313- 327

(65) Conway K, Russell G. Couple's grief and experience of support in the aftermath of miscarriage. Br J Med Psychol 2000; 73: 531- 545

(66) Najman JM, Vance JC, Boyle F, Embleton G, Foster B, Thearle J. The impact of a child death on marital adjustment. Soc Sci Med 1993; 37: 1005-10.

(67) RANDO T. Parental loss of a child. Champaign IL: Research Press, 1986

(68) Warland J. The midwife and the bereaved family. Ascot Vale, Victoria: Ausmed, 2000 
ORIGINALES Y REVISIONES

(69) CECIL R. Memories of pregnancy loss: recollections of elderly women in Northern Ireland. En Cecil R (ed): The anthropology of pregnancy loss. Oxford: Berg, 1996; pp 179-196

(70) LEON I. Choreographing grief on the obstetric unit. Am J Orthopsychiatry 1992; 62: 7-8

(71) RAPHAEL B. Preventive intervention with the recently bereaved. Arch Gen Psychiatry 1977; 34: $1450-1454$

(72) Leon I. Perinatal loss: a critique of current hospital practices. Clin Pediatr (Phila) 1992; 366374 